\title{
Localization of L-band RFI sources from SMAP data
}

\author{
Yan Soldo $*^{(1,2)}$, Paolo de Matthaeis ${ }^{(1,2)}$, and David M. Le Vine ${ }^{(1)}$ \\ (1) NASA Goddard Space Flight Center, Greenbelt, MD, USA \\ (2) GESTAR, Greenbelt, MD, USA
}

\begin{abstract}
RFI (Radio-Frequency Interference) in the 1400-1427 $\mathrm{MHz}$ band degrades the quality of measurements made by satellite missions such as SMAP (Soil Moisture Active/Passive), Aquarius and SMOS (Soil Moisture and Ocean Salinity). A technique is presented here to estimate the location on the ground of RFI sources using SMAP measurements. The results of this technique have been validated against data derived by other means.
\end{abstract}

\section{Introduction}

The presence of man-made emissions in the protected 1400-1427 $\mathrm{MHz}$ band contaminates the data from spaceborne radiometers that rely on this frequency to make passive measurements of Earth's natural radiation. Since the first observations of RFI (Radio-Frequency Interference) in this band, many studies have addressed the detection, localization and mitigation of RFI signals $[1,2]$.

An algorithm is presented here to localize the sources of RFI using data from SMAP (Soil Moisture Active/Passive), a satellite mission designed to map soil moisture worldwide and operating in the protected part of the L-band [3].

\section{Method}

The localization algorithm processes SMAP data from different orbits independently and uses the measurements of antenna temperatures before (called $\mathrm{T}_{\mathrm{A}}$ ) and after RFI

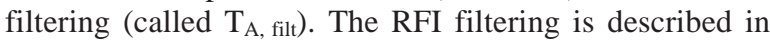
[4]. The algorithm is based on an iterative process and consists in the following steps.

\subsection{Estimation of the impact of RFI on the antenna temperatures}

At first, the algorithm estimates the impact of RFI on the measured antenna temperatures. This step relies on the antenna temperatures after RFI filtering $\left(\mathrm{T}_{\mathrm{A} \_ \text {filt }}\right)$ that are distributed in the SMAP products. In fact, the difference between $\mathrm{T}_{\mathrm{A}_{-} \text {filt }}$ and $\mathrm{T}_{\mathrm{A}}$ can be regarded as the impact of RFI as estimated by the RFI filtering algorithm.
However, two issues can arise. First, some measurements are so heavily affected by RFI that the filtering algorithm cannot provide an estimate of the RFI-free antenna temperature. Second, some level of RFI may not be detected correctly by the filtering algorithm and may affect the $\mathrm{T}_{\mathrm{A} \_ \text {filt }}$ as well.

The localization algorithm copes with the first issue by using the valid $\mathrm{T}_{\mathrm{A}_{-} \text {filt }}$ to fill the missing values using an interpolation. The second issue is partially addressed by comparing the $\mathrm{T}_{\mathrm{A}_{-} \text {filt }}$ against a fixed threshold (computed from a land emissivity model that overestimates the level of natural radiation) and discarding the $\mathrm{T}_{\mathrm{A}_{-} \text {filt }}$ above this threshold.

\subsection{Selection of contaminated measurements}

The second step consists in identifying the measurements that will be used for RFI localization. The selection criterion used here is critical because if too many measurements are selected it would become difficult to distinguish RFI sources close to one another; on the other hand, selecting too few measurements would mean not being able to localize weak RFI sources. To solve this problem an iterative approach was implemented that selects only a few measurements at first and then selects an increasing number of measurements at every iteration. This way the algorithm locates at first the strongest RFI and then it progressively identifies weaker RFI.

\subsection{Clustering}

Once the measurements have been selected, the algorithm attempts to organize them into clusters. This problem is well known in machine learning and several clustering algorithms have been developed over the years. In particular, the DBSCAN algorithm [5] has some properties that make it well suited for this application: it does not need to know the number of clusters a priori (the number of active RFI sources is not known a priori); it allows the presence of outliers (helps to account for false RFI detection); and does not need the input points to be in a regular grid.

Basically, DBSCAN creates a cluster if there is at least one input point with $N$ neighbors within a distance $d$, where $N$ and $d$ are input parameters. 


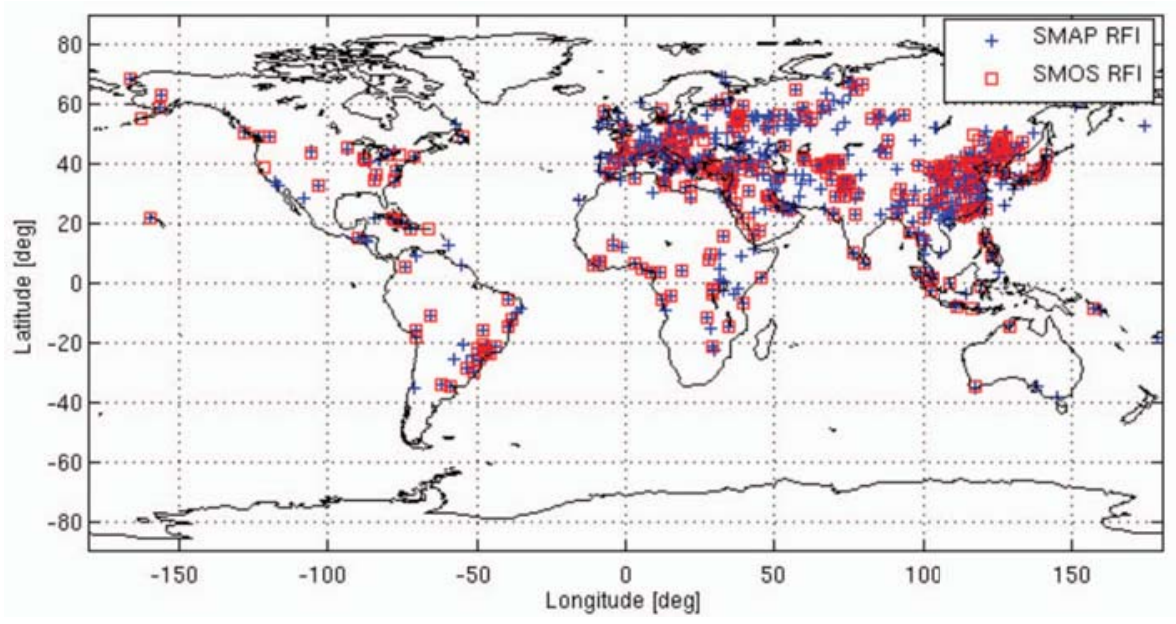

Figure 1. Map of SMOS and SMAP RFI localizations for the period June-August 2015

\subsection{Selection of clusters}

At this point the algorithm selects only the clusters that have low eccentricity and that are not adjacent to the swath edges. These criteria aim at discarding clusters that would potentially lead to less accurate localizations.

\subsection{Centroids}

Finally, the algorithm computes the centroid of each cluster. The centroids are computed as:

$$
\varphi_{c}=\frac{\sum_{i}^{N} \varphi_{i} T_{i}}{\sum_{i}^{N} T_{i}}
$$

where $\varphi$ is the geographic coordinate (latitude or longitude), $\mathrm{T}$ is antenna temperature due to the RFI (difference between $\mathrm{T}_{\mathrm{A}_{\text {filt }}}$ and $\mathrm{T}_{\mathrm{A}}$ ), the subscript $\mathrm{i}$ indicates the $\mathrm{i}$-th footprint of the cluster and the subscript $\mathrm{c}$ indicates the centroid.

\section{Results}

The localization dataset obtained by applying this technique has been compared against data derived by other means, such as:

- locations of RFI sources measured in situ with GPS (Global Positioning System) receivers;

- locations of DEW (Distant Early Warning) stations, that are known sources of RFI in this band;

- RFI localizations performed by SMOS (Soil Moisture Ocean Salinity), another satellite mission with a radiometer operating within the same bandwidth.

In particular, SMOS and SMAP localizations have been compared globally for the period June-August 2015 (see Figure 1).

\section{Conclusions}

In conclusion, the RFI localization provided by this algorithm agrees well with other relevant datasets, with an estimated error of less than 1-2 km in most cases. Also, the comparison with SMOS results has highlighted which types of RFI are more likely to be detected and localized correctly by SMAP (i.e., RFI near coastline or weak RFI) or by SMOS (i.e., RFI sources emitting sporadically).

\section{References}

1. D. M. Le Vine, P. de Matthaeis, C. S. Ruf and D. D. Chen, "Aquarius RFI Detection and Mitigation Algorithm: Assessment and Examples", IEEE Transactions on Geoscience and Remote Sensing, 52, 8, August 2014, pp. 4574-4584.

2. R. Oliva, E. Daganzo, P. Richaume, Y. Kerr, F. Cabot, Y. Soldo, E. Anterrieu, N. Reul, A. Gutierrez, J. Barbosa, G. Lopes, "Status of Radio Frequency Interference (RFI) in the 1400-1427MHz passive band based on six years of SMOS mission," Remote Sensing of Environment, 180, July 2016, pp. 64-75.

3. J. R. Piepmeier, J. T. Johnson, P. N. Mohammed, D. Bradley, C. Ruf, M. Aksoy, R. Garcia, D. Hudson, L. Miles, M. Wong "Radio-frequency interference mitigation for the soil moisture active passive microwave radiometer," IEEE Transactions on Geoscience and Remote Sensing, 52, 1, January 2014, pp. 761-775.

4. D. Entekhabi, E. G. Njoku, P. E. O'Neill, K. H. Kellogg, W. T. Crow, W. N. Edelstein, J. K. Entin, S. D. Goodman, T. J. Jackson, J. Johnson, J. Kimball, "The soil moisture active passive (SMAP) mission," Proceedings of the IEEE, 98, 5, May 2014, pp. 704-716. 
5. M. Ester, H. P. Kriegel, J. Sander, X. Xu, “A densitybased algorithm for discovering clusters in large spatial databases with noise," Kdd, 96, 34, August 1996, pp. 226-

231. 